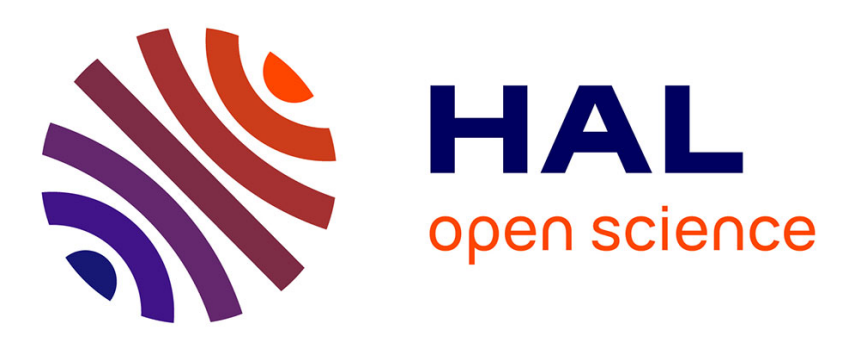

\title{
The Evolution of Variability and Robustness in Neural Development
}

\author{
P. Robin Hiesinger, Bassem A Hassan
}

\section{To cite this version:}

P. Robin Hiesinger, Bassem A Hassan. The Evolution of Variability and Robustness in Neural Development. Trends in Neurosciences, In press, 10.1016/j.tins.2018.05.007 . hal-01826196

\section{HAL Id: hal-01826196 \\ https://hal.sorbonne-universite.fr/hal-01826196}

Submitted on 29 Jun 2018

HAL is a multi-disciplinary open access archive for the deposit and dissemination of scientific research documents, whether they are published or not. The documents may come from teaching and research institutions in France or abroad, or from public or private research centers.
L'archive ouverte pluridisciplinaire HAL, est destinée au dépôt et à la diffusion de documents scientifiques de niveau recherche, publiés ou non, émanant des établissements d'enseignement et de recherche français ou étrangers, des laboratoires publics ou privés. 


\section{The Evolution of Variability and Robustness in Neural Development}

\section{P. Robin Hiesinger ${ }^{1, \star}$ and Bassem A. Hassan ${ }^{1,2, \star}$}

As in all biological systems, neurons and their networks must balance precision with variability. Phenotypic precision and phenotypic variability can both occur with remarkable robustness, where robustness is defined as the ability to tolerate perturbation. Variability in genotype-phenotype mapping produces phenotypic variability despite identical genetic information. The resulting variability among genetically identical neurons can contribute to the robustness of brain development. Similarly, variability of genetically identical individuals can contribute to evolutionary robustness. We discuss here shared principles of developmental robustness and evolutionary robustness, and highlight scenarios where such principles result in neural networks that achieve robustness of precision or variability.

From Variability to Robustness in Brain Development and Evolution

Precision in neuronal differentiation and connectivity is a remarkable outcome of neural development. Neuron subtypes, their ratios, distribution, and synaptic connections are all highly reproducible. However, the outcome of neural development is, in fact, not always precise. Examples abound of genetically identical individuals with variable nervous systems, from worms to humans. For instance, genetically identical $C$. elegans or grasshoppers exhibit anatomical differences in their connectivity that were already described in the 1970s [1,2]. The brains of monozygotic human twins also show considerable structural differences [3], and it remains unclear to what extent this is due to environmental factors or inherently imprecise development. Similarly, peripheral nervous system innervation of the left and right sides of human fetuses exhibit differences that have been attributed to inherently imprecise development [4]. These observations showcase variability despite identical genes, suggesting that nonheritable factors play important roles in generating variability during neuronal development.

The outcome of neural development is selected not only for optimized function but also for robustness. The robustness of a system is defined as its ability to tolerate perturbation (Table 1). Imagine, for instance, an enzyme that functions optimally but loses all activity if any of its amino acids are changed by mutation. By contrast, imagine another enzyme that is somewhat less efficient but can tolerate the majority of single amino acid exchanges without significant reduction in its activity. In any species with a mutation rate higher than zero, the latter enzyme has a selective advantage because it is more robust. Robustness to excessive variation may contribute to the evolvability of biological systems. This is because robustness allows the accumulation of mutations (cryptic genetic variation [5]), and thus eventually 'evolutionary innovation', with minimal effects on fitness in the intervening time (reviewed in [6-8]), although the generality of this idea has been questioned [9]. Importantly, the robustness of the phenotype of an organism to perturbation is due in large part to developmental processes that can tolerate random genetic mutations and unpredictable environmental perturbations. This has historically been described as 'canalization of development' [10]. The relationship between

\section{Highlights}

Identical genes do not ensure identical processes during neural development, partly owing to the intrinsic noise in molecular processes.

Noisy neural development may result in either invariable or variable adult structure and function.

Developmental noise and variability contribute to robustness to perturbation.

Evolutionary robustness and developmental robustness share some of the same principles.

${ }^{1}$ Division of Neurobiology, Institute for Biology, Freie Universität Berlin, 14195 Berlin, Germany ${ }^{2}$ Institut du Cerveau et de la Moelle Epinière (ICM), Hôpital Pitié-

Salpêtrière, Université Pierre et Marie Curie (UPMC), Sorbonne Universités, Institut National de la Santé et de la Recherche Médicale (INSERM), Centre National de la Recherche Scientifique (CNRS), Paris, France

${ }^{*}$ Correspondence: robin.hiesinger@fu-berlin.de (P.R. Hiesinger) and bassem.hassan@icm-institute.org (B.A. Hassan). 
Table 1. Operational Definitions and Examples for Key Terms

\begin{tabular}{|c|c|c|}
\hline Term & Definition & Examples \\
\hline Stochastic (or 'truly random') process & $\begin{array}{l}\text { A process in which at any point in time the precise } \\
\text { state or value is (i) unpredictable and neither depends } \\
\text { on (ii) previous values or states of the system, nor on } \\
\text { (iii) an inherent bias of the system. We use the term } \\
\text { stochastic synonymously with random. }\end{array}$ & $\begin{array}{l}\text { Dice roll. For each roll of a 'fair' dice, the probability of each of } \\
\text { the six outcomes is exactly } 1 / 6 \text { and does not depend on } \\
\text { previous throws of the dice. } \\
\text { The meiotic segregation of chromosomes. The distribution of } X \\
\text { and } Y \text { chromosomes is } 1 / 2 \text { and does not depend on previous } \\
\text { 'drawings'. Notably, there are factors that bias this outcome } \\
\text { and there remains doubt as to what extent biological } \\
\text { processes in general are truly stochastic. See noisy process. }\end{array}$ \\
\hline $\begin{array}{l}\text { Noisy (imprecise, non-deterministic } \\
\text { or probabilistic) process }\end{array}$ & $\begin{array}{l}\text { We define as 'noisy' a process in which at any point in } \\
\text { time the precise state or value is unpredictable. } \\
\text { However, in contrast to a truly random process, there } \\
\text { might be a dependency on previous states of the } \\
\text { system or an inherent bias. We use noisy } \\
\text { synonymously with imprecise, non-deterministic, and } \\
\text { probabilistic. }\end{array}$ & $\begin{array}{l}\text { Growth cone filopodia extension/retraction dynamics are } \\
\text { unpredictable, but are typically biased towards the 'leading } \\
\text { edge'. } \\
\text { Dscam splice variants are non-predictable, but may not have } \\
\text { equal probability for all variants. }\end{array}$ \\
\hline Deterministic process & $\begin{array}{l}\text { A process in which any future state or value of the } \\
\text { system is precisely predictable. Hence, a deterministic } \\
\text { process always produces the same outcome. }\end{array}$ & $\begin{array}{l}\text { Transcription of the same DNA sequence will deterministically } \\
\text { yield the same RNA sequence, given identical conditions and } \\
\text { no error rate. To the extent that the error rate increases, the } \\
\text { process can become noisy. Note that a deterministic outcome } \\
\text { depends on the exact state or value under consideration: given } \\
\text { a large number of dice throws, the average outcome will } \\
\text { deterministically converge to } 3.5 \text {. Two sister cells may } \\
\text { stochastically attain either fate A or B, but the process can } \\
\text { deterministically ensure that exactly one cell becomes A and } \\
\text { one cell becomes B. }\end{array}$ \\
\hline Variable outcome & $\begin{array}{l}\text { We define the outcome of process as variable if that } \\
\text { outcome is unpredictable and different when } \\
\text { repeated. }\end{array}$ & $\begin{array}{l}\text { Variability can originate from stochastic or noisy processes. } \\
\text { Rolling a dice leads to variable outcomes that are stochastically } \\
\text { distributed }(1,2,3,4,5,6) \text {. Noisy growth cone filopodia } \\
\text { dynamics lead to variability in the direction of growth. }\end{array}$ \\
\hline Robust (or canalized) outcome & $\begin{array}{l}\text { Robustness is the property of tolerating perturbation. } \\
\text { Both precision or variability can be robust. We use } \\
\text { robustness of precision synonymously with } \\
\text { Waddington's canalization. }\end{array}$ & $\begin{array}{l}\text { The precise expression or function of a protein can be robust to } \\
\text { mutation, in other words many (possibly most) mutations only } \\
\text { quantitatively change expression or function levels. These } \\
\text { changes may be subthreshold with respect to its } \\
\text { consequences or gradually affect a higher-order process. } \\
\text { Dscam splicing in neurons and V(D)J recombination in immune } \\
\text { cells create variability. The variability as such can be robust to } \\
\text { genetic or environmental perturbation. Waddington's classic } \\
\text { concept of canalization is restricted to robustness of precision, } \\
\text { in other words constancy of outcome in the wild type, which is } \\
\text { often reduced in Drosophila mutants. }\end{array}$ \\
\hline
\end{tabular}

developmental processes and phenotypic robustness is often difficult to describe because of nonlinearities inherent in the molecular and cellular processes underlying development [11].

Evolutionary robustness requires variability among individuals. Individuals are the outcome of development, and this outcome can be variable or precise, and both can be robustly so (i.e., both variability and precision among individuals in the population can be robust to perturbation). Similarly, developmental processes can be deterministic or noisy, and both robustly so (Table 1). However, invariant outcomes do not necessarily require deterministic developmental mechanisms. As we will discuss in the first section, some neural network features may need to be precise, but the developmental processes generating them may need to be noisy to achieve that precision. In this case, variability among neurons may be crucial to achieve developmental robustness, similarly to how variability among individuals is crucial for evolutionary robustness. 
Therefore, in both developmental and evolutionary contexts, noise in a preceding step can lead either to a precise or to a variable outcome in the subsequent step.

The differences between monozygotic twins, and between left and right peripheral innervation patterns, suggest that noisy development underlies variable outcomes even in the absence of germline mutations. Hence, developmental processes can be noisy as a result of genetic or non-genetic causes. Phenotypic variability of circuit function owing to heritable genetic differences is subject to evolutionary selection. However, can phenotypic variability result from identical genes and noisy development, including somatic, non-heritable genetic differences? In the second section of this Opinion we discuss how variability itself might be evolutionarily selectable during neural development.

In the following two sections we therefore ask two questions. First, how does phenotypic variability in neural development arise even when genes are identical? Second, what are the possible evolutionary consequences of this type of phenotypic variability?

\section{Developmental Sources of Variability Despite Identical Genes}

Intrinsically noisy developmental processes can lead to either variability or precision in the outcome. We have previously discussed the contribution of genetically encoded noisy developmental processes in brain wiring [12]. Our core argument was that synapse-specific brain wiring is achievable through molecular functions that not only tolerate noise but actively utilize it. Prominent examples include dendritic self-avoidance based on stochastic filopodial growth and noisy splicing of a cell adhesion molecule $[13,14]$. Mechanisms based on noise may be part of a multistep developmental process that also includes molecular functions, such as synaptic matchmaking, that do not utilize noise but must tolerate it. Roger Sperry's influential chemoaffinity theory [15] is the foundational framework for the latter type of molecular functions. Chemoaffinity has since then been extended by many elegant studies that revealed functions of 'connectivity molecules' that function as 'Sperry molecules' to various degrees [16-18]. By contrast, a framework of rules focuses on the principle that is executed through molecular actions [12]. Importantly, developmental rules (such as self-avoidance or lateral inhibition) govern both noisy (as opposed to only 'noise-tolerating') and deterministic molecular mechanisms, exactly as physical laws govern both noisy and deterministic behaviors of matter. To understand the evolution of variability and robustness, we need to understand developmental rules that operate on noisy molecular mechanisms and cellular dynamics.

Phenotypic outcomes can be highly precise not only as a result of deterministic processes but can also be due to intrinsically stochastic processes. A good example of the latter is the precisely controlled number of neurons in C. elegans, where exactly 302 neurons result from developmental cell competition that does not allow the prediction of which cells will die in the process [19]. Similarly, photoreceptor neurons in the fly eye differentiate following a nondeterministic cell-sorting process that does not allow one to predict which cells will become neurons and which cells will die; nonetheless, the number of cells of a single eye facet (called an ommatidium) is invariable, and the precision of optical axes within and across ommatidia is astounding [20]. Thus, when does developmental noise lead to invariable phenotypes, and when does it lead to phenotypic variability?

First, we need to consider that neural circuits, like any biological structure, grow based on algorithmic information. An algorithm is defined as a series of steps where the output of preceding steps becomes the input of the following step. Algorithmic information, as opposed to total endpoint information (i.e., blueprints), is defined by the rules applied at each step, and 
not by the precise input/output values. This is the basis for developmental flexibility. During fly eye development, for instance, half the cells can be continuously experimentally killed through continued expression of an apoptosis inducer in progenitor cells. Remarkably, a morphologically normal eye and precise eye-brain wiring develop, and this technique is routinely used in fly laboratories to generate homozygous mutant eyes [21,22]. This is because, during eye development, stochastic recruitment and cell killing are an integral part of the rules. We note that the noisy, flexible nature of eye development also manifests itself in apparent genetic redundancy: two mutations that independently increase cell death or reduce the accuracy of patterned cell sorting through different mechanisms may result in a disrupted outcome, while each mutation alone may lie within the margins of what noisy, flexible development can still compensate for.

Algorithmic growth not only accommodates stochastic processes but can even make such processes an integral part of the algorithmic rules [12]. The extent to which variability in phenotypic outcome is under evolutionary selection can determine the extent to which noisy development is 'preserved'. In the case of the fly, the optical axes between the individual single eye facets (ommatidia) of the compound eye and the precision of the underlying wiring of photoreceptor axons in the brain must be precise for spatial vision to function. By contrast, the precise number of ommatidia per complex eye is variable and is likely under much less selective pressure. Hence, the same underlying noisy growth leads not only to astonishing precision in the geometry of the fly eye and the underlying brain wiring pattern, but also to a significantly variable number of component eye facets between individual flies. Perhaps even more remarkably, the sizes of the left and right eyes of the same fly, while highly correlated, are nonetheless not identical [23].

Another aspect to consider is that, in many respects, individual neurons operate as autonomous agents during neural development. Neurons grow axonal growth cones and dendritic branches through local decision-making processes in an unknown environment. Each branch of the dendritic tree grows without knowledge of a branch at the other end of the tree, let alone the branches of other trees. Similarly, the axonal growth cone individually responds to what it encounters in the environment. This allows local decisions in the face of an obstacle, loss of branches, and imprecision in the molecular environment. Each of the autonomously acting agents can be considered to be a player in a game with set rules. Each player makes individual, noisy, and local decisions, even in genetically identical left and right brain hemispheres, but following the same rules.

To illustrate this point, consider molecule families such as the highly variable Drosophila Dscams and vertebrate protocadherins. For both types of molecules specific isoforms are expressed noisily in a cell-autonomous fashion, such that only a few of potentially thousands of possible variants are expressed in each neuron. This variable identity allows a neuron to distinguish self from non-self. Self-avoidance based on noisy filopodial extension dynamics and neuronal dendrite recognition of self leads to spread-out growth of its dendritic tree [13,14]. We would argue that the functionally significant dendrite spreading is under evolutionary selection, while the precise location of individual branching points is not. Dendrite spreading is based on a developmental rule-set consisting of iterated noisy filopodial growth, self-avoidance, and branching. The process leads to robustness of stereotypically spread-out dendritic trees (which are functionally important), but variability in the precise branching pattern (which is not). In fact, Dscam and protocadherins are not only examples of molecules that execute developmental rules based on noisy processes in an algorithmic fashion, but are also prime examples of evolutionary selection for variability per se, as discussed in the second section of this article. 
In contrast to Dscam and protocadherins, several cell adhesion molecules have been shown to function with remarkable specificity in the matchmaking of specific synaptic partners, including sidekick-2 [24] in the mouse retina, teneurins in the fly olfactory system [25], and Syg-1/Syg-2 in a $C$. elegans motor neuron [26]. Interestingly, none of these mutants affect the ability of a neuron to make synapses per se, and in the absence of each of these molecules synapses are formed in varying numbers with other, often incorrect, partners. We would argue that these are examples of molecular adhesion that bias the probabilistic nature of synapse formation - a developmental rule based on a noisy process. In terms of algorithmic growth, this biasing typically occurs towards the end of the synaptic specification process. Interestingly, sets of molecules have been identified in gene expression profiles that are sufficient to predict synaptic partners in C. elegans [27,28] and Drosophila [29]. While it is tempting to interpret these molecules in terms of 'lock and key' for a synaptic code, genes that computationally predict partnerships may not function as part of the developmental algorithm at all, in the same way as the expression of a neurotransmitter in a presynaptic cell and its receptor in postsynaptic cell does not dictate their connectivity.

Is noisy development really essential to create robustness in the outcome? As discussed above, dendritic growth based on self-avoidance is robust because of (i) noisy filopodial exploration, and (ii) local decisions of dendrites when to 'self-avoid'. However, at least in theory, robustness could still be achieved with deterministic filopodial exploration, but not without local decision-making, as discussed below. We must therefore consider how a deterministic model could work, and when noisy development may be advantageous or even be required for robustness (Figure $1 \mathrm{~A}-\mathrm{C}$ ).

Biological examples show that stochastic filopodial dynamics provide a substrate for synaptotropic growth towards a brain region with more synaptic partners; similarly, stochastic filopodial dynamics provide the substrate for self-avoidance to generate dendrite spreading [30-32]. In theory, however, a deterministic filopodial 'probing program' could work, provided that it probed all possible directions evenly, by searching every $N$ degrees rotationally in a $360^{\circ}$ circle (Figure 1C). Provided that the growing dendritic tree can operate as an autonomous agent, such a theoretical deterministic behavior would allow similar flexibility when hitting obstacles or during growth towards regions with increased synaptic partner availability. However, we are not aware of neuronal examples where deterministic axonal or dendritic filopodial probing of an unknown environment occurs. On the other hand, examples abound of axonal and dendritic growth based on non-deterministic, flexible, and robust development [33-35]. An interesting question concerns what is 'easier' to encode - in terms of information cost - and what is more robust in the face of perturbation through mutation: a deterministic algorithm or an algorithm that employs intrinsically stochastic processes?

Arguably, random exploration is the ideal way for an axon or dendrite to evenly explore an unknown environment. Any deterministic algorithm designed to both comprehensively and evenly explore the environment may come close to, but never fully achieve, the random ideal. If intrinsically noisy molecular processes create random exploration, this may be less costly to genetically encode than a deterministic program that ensures both even and complete exploration (Figure 1). A random process creates a precise Gaussian distribution around the mean, 'majority' behavior (Figure 1A,B). The importance of the width of this distribution (i.e., noisiness) becomes clear when a selection pressure or perturbation is applied. Consider the case of an exploring axonal or dendritic arbor whose target may be closer or further away. If filopodial exploration is precise (the distribution narrow), the likelihood of missing the target increases (Figure 1A). By contrast, a noisier (wider) random distribution will increase the 
(A)

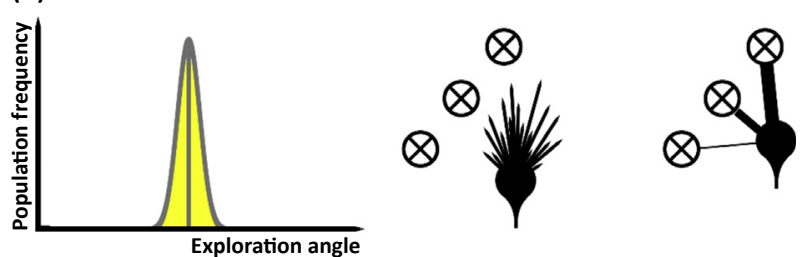

(B)
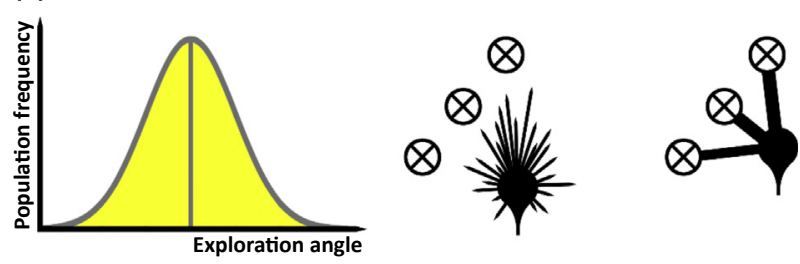

(C)
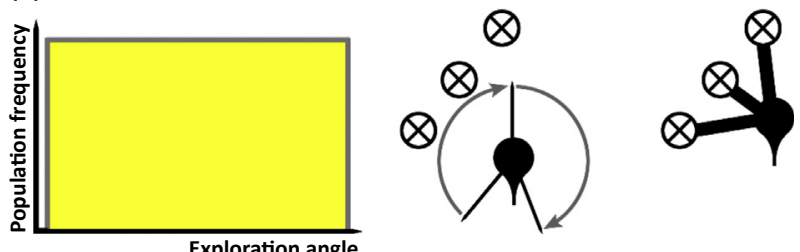

Trends in Neurosciences

Figure 1. Developmental Robustness. (A) Directed growth of axonal or dendritic arbors may occur with little random filopodial exploration deviating from the mean. The filopodial distribution is narrow, the cost is low (yellow area), and the probability of identifying a target deviating from the mean is reduced (black connection bars on the right). (B) Directed growth with a wide random distribution of filopodial exploration. The cost is higher (yellow area) and the probability of identifying a target deviating from the mean is high. (C) Directed growth with deterministic 'circular exploration'. The cost is highest (yellow area) and the probability of identifying a target deviating from the mean is not much higher than in (B).

probability of finding the target (Figure 1B). Increasing this probability through a deterministic process may come at a much higher cost (Figure 1C). Hence, there are good theoretical reasons why, and empirical examples of how, (neuro-)developmental processes utilize noise to minimize cost and create robustness in the face of uncertainty or perturbation.

Lastly, an important question to consider in this context is: what is the source and nature of noise during neural development? An obvious source of noise is apparent at the molecular level. Molecular noise can be defined as 'stochastic fluctuations inherent to biochemical processes due to the discreteness of molecules and their random collision' [36]. For example, molecular noise is an essential feature of the bacterial chemotaxis network, and explains individually variable behavior in bacteria [37]. In addition, somatic genetic mutations during development can create clones of cells that are genetically different from each other. For instance, human monozygotic twins show somatic genetic alterations such as copy-number variation $[38,39]$ as well as epigenetic changes such as DNA methylation and histone acetylation [40]. These somatic genetic differences are stochastic, non-heritable by nature, and can create phenotypic differences such as disease susceptibility or distinct behavioral personality traits. Mechanisms that create somatic genetic diversity, such as transposable element mobilization, are highly active in the human brain, as well as in the brains of other species including insects and rodents $[41,42]$. The retrotransposon LINE-1 alone may have the potential to render every neuron in a mammalian brain genomically unique [43]. 
In summary, identical genes do not encode identical processes or outcomes during neural development. Noisy developmental processes can change both the genome of each individual cell as well as the behavior of two genetically identical neurons. Noise thereby contributes to both variability and robustness in the developmental outcome. In the next section we discuss how phenotypic variability in neural development can be an evolutionarily selectable property.

\section{Evolution of Robustness, Robustness of Evolution}

We have thus far argued that intrinsically noisy developmental processes can contribute to both robustly variable and invariable phenotypic outcomes. Noisy processes actively facilitate robustness in the contexts of algorithmic development and neurons that operate as 'autonomous agents' during development. The evolutionary selection of a noisy process is therefore plausible if robustness is advantageous. Robustness of invariable outcomes is relatively easy to understand: if a developmental process can achieve, for instance, a precise fly eye anatomy and visual map wiring even though half of the cells may die during development, then this is an advantageous, and selectable, type of robustness.

Phenotypic variability is similarly easy to understand if selection of individual phenotypic variants means selection of genotypic variants. However, as we have seen, variable phenotypic outcomes can also result from identical genes, based on intrinsically noisy developmental processes. From an evolutionary perspective, only genetically encoded variants are selectable. Individual phenotypic variants resulting from intrinsically noisy development of identical genomes are invisible to the all-seeing eye of natural selection because they are not heritable. Or are they?

Phenotypic variability has been investigated in genetically identical individuals of species that reproduce through parthenogenesis, for example the grasshopper [1] and the clonal marbled crayfish [44]. Isogenic batch-mates of this clonal crayfish reared under identical conditions exhibit a large degree of variation in coloration, growth, lifespan, reproduction, behavior, and number of sense organs. The fact that variation was also found with respect to fluctuating asymmetry strongly indicates the intrinsically stochastic developmental component of the phenotype of an individual. Strikingly, the marbled crayfish is a highly robust, highly fecund, and fast-spreading species that appears to adapt well to a variety of ecological niches.

In clonal species, the selective advantage of phenotypic variability is obvious: if selection pressure for (or against) some specific phenotypic variant increases, other variants with the same genome survive. However, the observation that the same genotype can produce different phenotypes (even under identical environmental conditions) implies that different genotypes can produce overlapping phenotypes. As shown in Figure 2, if genotype-phenotype mapping is precise (the distribution is narrow), a single mutation (from genotype $A$ to genotype $B$ in Figure 2) will result in failure for all individuals (be it neurons or animals). By contrast, a noisier (wider) stochastic distribution will allow a certain (sometimes precise!) number of individuals to succeed. A simple hypothetical illustration of this would be two mice where one has a promoter mutation that lowers the isoform variability of a protocadherin, leading to faulty dendritic selfavoidance. Because the rules that govern these developmental events utilize noisy processes, and are robust by their very nature, these manifestly different genomes can nonetheless each generate a distribution of phenotypes, some of which are indistinguishable (Figure 2). Thus, even under strong selection pressure, more than one of these genotypes can give rise to a phenotype that enhances fitness and will thus pass a selection bottleneck (Figure 2). Therefore, at least a degree of genetic diversity will be maintained, and the population can in principle recover faster than would be expected if a given genotype only gives rise to one phenotype. The 


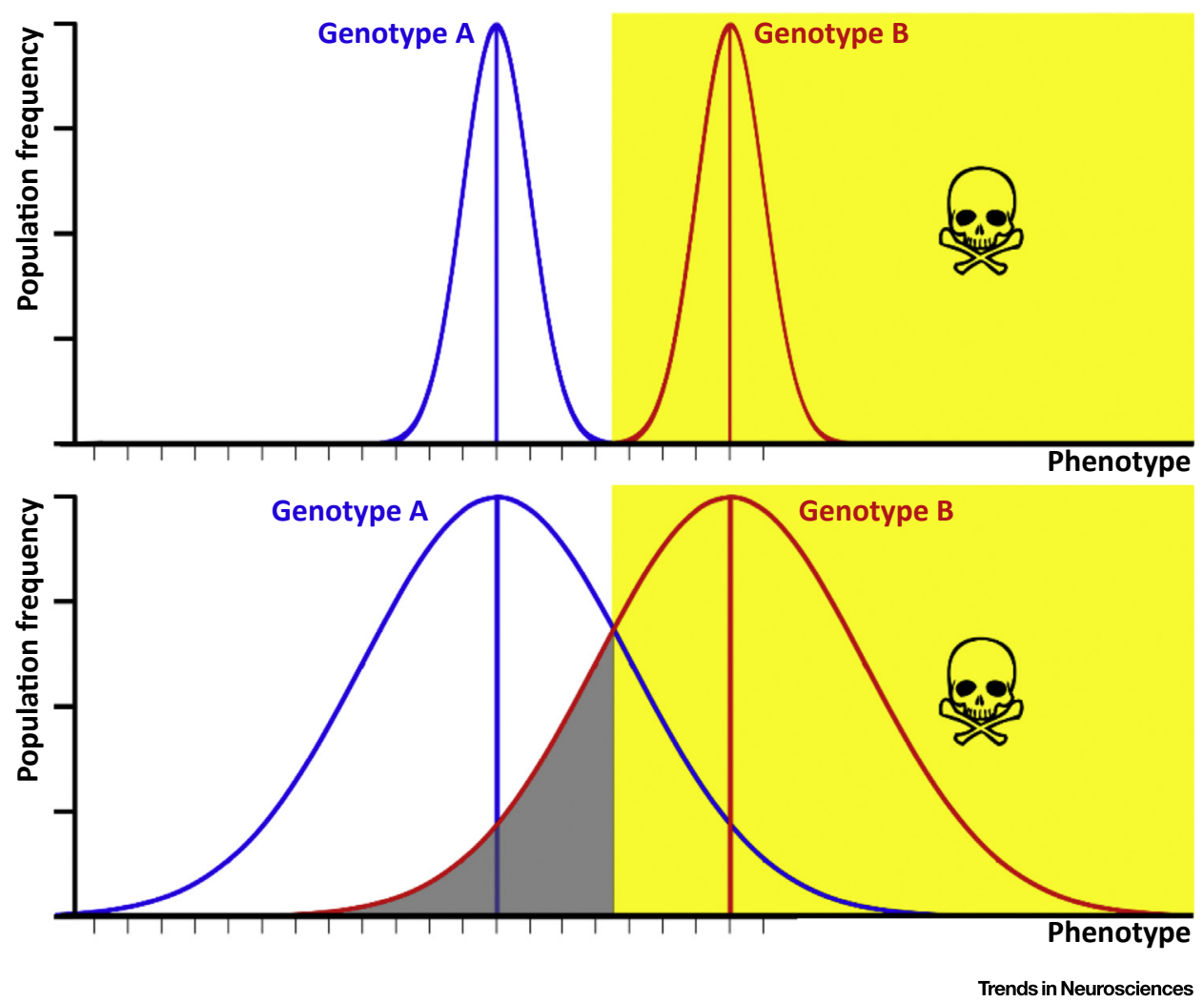

Figure 2. Evolutionary Robustness. (Top graph) Two genotypes A and B with precise genotype-phenotype mapping. (Bottom graph) The same two genotypes with noisy genotype-phenotype mapping. Greater variation (wider distributions) around the same mean may allow more individuals (grey area under red curve) to survive selection pressure (yellow area).

advent of CRISPR-mediated genome-editing technology should allow formal testing of this idea in genetically identical animals. If this hypothesis is correct, then CRISPR-mediated genome editing of a given naturally occurring allele to another should shift the mean population value of a phenotype under the control of the edited gene, but there will still be individuals who have the same phenotype while carrying different alleles.

A study of individual left versus right walking preference in Drosophila demonstrates that preference variability - rather than the actual walking preference - is the heritable trait [45]. Specifically, the authors found that individual flies repeatedly preferred to walk to the right or to the left - or showed no preference - when given a free choice in a Y-maze. The population thus displayed variation across a handedness-preference mean. When 'left-handed' (or 'righthanded') flies were crossed with each other, the offspring regenerated the entire range of handedness behavior, with no change in the mean handedness of the population. Remarkably, QTL mapping identified a developmental synaptic connectivity gene whose function affects the variability of walking handedness across the population, but not the mean population performance. What is perhaps more remarkable is that the gene identified as a locus of handedness variability - encoding a protein of the teneurin family - is often cited as a prime example of a molecule that mediates invariable and deterministic synaptic matching in the fly olfactory system [25]. Interestingly, a recent study provided a quantitative characterization of wiring variability in the fly olfactory system [46]. The authors identified imprecisions due to inherently 
noisy development (which they call 'noise') or due to plastic changes (e.g., homeostatic compensation, which they call 'signal'). However, as discussed here, we note that such variability in the outcome may in some cases also be advantageous for the fly or the population.

Similarly, another recent study provides an exquisite example of an intrinsically noisy neurodevelopmental process in Drosophila in which both variable and invariant outcomes co-occur [47]. In this case, the same progenitor cells can produce motor neurons predictably with high precision and glial cells with high variability. The authors make a strong case that this coordinated 'hard wired motor neuron generation and flexible glia production' contributes to the robust development and evolution of neural circuitry. In sum, while it is obviously true that the individual variants resulting from non-genetic variation are not selectable, variability itself is. This idea has also been formulated as a bet-hedging strategy: the same genotype 'bets' on possible selection pressure with a distribution of phenotypes [48]. From the perspective of neural development, intrinsically stochastic processes can produce a wide range of phenotypically selectable variability in circuit connectivity and behavior from a narrower range of pre-existing genetic variants. Hence, individuals from more genotypes can be successful if non-genetic variation is larger during development.

\section{Concluding Remarks}

We have discussed how noise, variability, and robustness are intimately linked in neural development, exactly as they are in evolution (see Outstanding Questions). Specifically, reproducible precision can be the outcome of noisy development in particular when robustness is a selective advantage (first question). Conversely, the genotype-phenotype map will allow for variability if that variability itself represents a selective advantage (second question). Hence, both noisy development and variability in the outcome can contribute in different ways to evolutionary diversity (third question).

Our discussion draws an analogy between developmental and evolutionary robustness. In the example summarized in Figure 1, larger variation in filopodial exploration leads to increased 'wiring success'. In this example, the noisy entities are the filopodia and the robust outcome is dendritic growth [31]. At a level higher, the variable entity can be neurons and the robust outcome is network behavior, as shown for rhythms in small neural circuits, for example [49]. In the case of phenotypic variability of neural circuits and animal behavior, the robust outcome is population behavior (Figure 2). As such, neural development may be no different from other developmental processes, but it is the variability and robustness of neural development that most profoundly influence personality! Thus, as Jean-Jacques Kupiec argues [50] - much as random genetic mutation constrained by natural selection underlies the origin of species molecular noise, including random somatic mutations, constrained by simple developmental rules, may underlie the origin of individuals.

\section{Acknowledgments}

We thank Drs Sophie Armitage, Jens Rolff, Ursula Habenicht, and Ariane Ramaekers for comments on the manuscript and all members of the laboratories of P.R.H. and B.A.H for discussion. This work was supported by an Einstein BIH (Berlin Institute of Health) Fellowship to B.A.H. Work in the Laboratory of P.R.H. is supported by grants from the Deutsche Forschungsgemeinschaft (DFG; grants SFB958 and SFB/TRR186) and the National Institutes of Health (NIH; RO1EY018884). Work in the laboratory of B.A.H. is supported by the program 'Investissements d'Avenir' (ANR-10IAIHU-06), the ICM, and an Allen Distinguished Investigator award from the Paul G. Allen Frontiers Group.

\section{References}

1. Goodman, C.S. (1978) Isogenic grasshoppers: genetic variability in the morphology of identified neurons. J. Comp. Neurol. 182, 681-705

\section{Outstanding Questions}

To what extent is reproducible precision in neural circuits the result of noisy development?

How precise is genotype-phenotype mapping for brain wiring; in other words, how variable can neural circuit wiring be given identical genes?

To what extent does variability based on identical genes contribute to evolutionary diversity? 
3. Mohr, A. et al. (2004) The similarity of brain morphology in healthy monozygotic twins. Brain Res. Cogn. Brain Res. 20, 106-110

4. Belle, M. et al. (2017) Tridimensional visualization and analysis of early human development. Cell 169, 161-173

5. Gibson, G. and Dworkin, I. (2004) Uncovering cryptic genetic variation. Nat. Rev. Genet. 5, 681-690

6. Felix, M.A. and Barkoulas, M. (2015) Pervasive robustness in biological systems. Nat. Rev. Genet. 16, 483-496

7. Wagner, A. (2008) Robustness and evolvability: a paradox resolved. Proc. Biol. Sci. 275, 91-100

8. Masel, J. and Trotter, M.V. (2010) Robustness and evolvability. Trends Genet. 26, 406-414

9. Mayer, C. and Hansen, T.F. (2017) Evolvability and robustness: a paradox restored. J. Theor. Biol. 430, 78-85

10. Waddington, C.H. (1942) Canalization of development and the inheritance of acquired characters. Nature 563-565

11. Beck, C.R. et al. (2010) LINE-1 retrotransposition activity in human genomes. Cell 141, 1159-1170

12. Hassan, B.A. and Hiesinger, P.R. (2015) Beyond molecular codes: simple rules to wire complex brains. Cell 163, 285-291

13. Kise, Y. and Schmucker, D. (2013) Role of self-avoidance in neuronal wiring. Curr. Opin. Neurobiol. 23, 983-989

14. Zipursky, S.L. and Grueber, W.B. (2013) The molecular basis of self-avoidance. Annu. Rev. Neurosci. 36, 547-568

15. Sperry, R.W. (1963) Chemoaffinity in the orderly growth of nerve fiber patterns and connections. Proc. Natl. Acad. Sci. U. S. A. 50, 703-710

16. Petrovic, M. and Schmucker, D. (2015) Axonal wiring in neural development: target-independent mechanisms help to establish precision and complexity. Bioessays 37, 996-1004

17. Zipursky, S.L. and Sanes, J.R. (2010) Chemoaffinity revisited: dscams, protocadherins, and neural circuit assembly. Cell 143, 343-353

18. de Wit, J. and Ghosh, A. (2016) Specification of synaptic connectivity by cell surface interactions. Nat. Rev. Neurosci. $17,22-35$

19. White, J.G. et al. (1986) The structure of the nervous system of the nematode Caenorhabditis elegans. Philos. Trans. R. Soc. Lond. B Biol. Sci. 314, 1-340

20. Kumar, J.P. (2012) Building an ommatidium one cell at a time. Dev. Dyn. 241, 136-149

21. Newsome, T.P. et al. (2000) Analysis of Drosophila photoreceptor axon guidance in eye-specific mosaics. Development 127 $851-860$

22. Stowers, R.S. and Schwarz, T.L. (1999) A genetic method for generating Drosophila eyes composed exclusively of mitotic clones of a single genotype. Genetics 152, 1631-1639

23. Vollmer, J. et al. (2016) A quantitative analysis of growth control in the Drosophila eye disc. Development 143, 1482-1490

24. Krishnaswamy, A. et al. (2015) Sidekick 2 directs formation of a retinal circuit that detects differential motion. Nature 524 , 466-470

25. Hong, W. et al. (2012) Teneurins instruct synaptic partner matching in an olfactory map. Nature 484, 201-207

26. Shen, K. et al. (2004) Synaptic specificity is generated by the synaptic guidepost protein SYG-2 and its receptor, SYG-1. Cell $116,869-881$

27. Baruch, L. et al. (2008) Using expression profiles of Caenorhabditis elegans neurons to identify genes that mediate synaptic connectivity. PLoS Comput. Biol. 4, e1000120
28. Kaufman, A. et al. (2006) Gene expression of Caenorhabditis elegans neurons carries information on their synaptic connectiv ity. PLoS Comput. Biol. 2, e167

29. Li, H. et al. (2017) Classifying Drosophila olfactory projection neuron subtypes by single-cell RNA sequencing. Cell 171 , 1206-1220

30. Vaughn, J.E. (1989) Fine structure of synaptogenesis in the vertebrate central nervous system. Synapse 3, 255-285

31. Niell, C.M. (2006) Theoretical analysis of a synaptotropic dendrite growth mechanism. J. Theor. Biol. 241, 39-48

32. Niell, C.M. et al. (2004) In vivo imaging of synapse formation on a growing dendritic arbor. Nat. Neurosci. 7, 254-260

33. Zschatzsch, M. et al. (2014) Regulation of branching dynamics by axon-intrinsic asymmetries in tyrosine kinase receptor signaling Elife 3, e01699

34. Ryglewski, S. et al. (2017) Intra-neuronal competition for synaptic partners conserves the amount of dendritic building material. Neuron 93, 632-645

35. Ozel, M.N. et al. (2015) Filopodial dynamics and growth cone stabilization in Drosophila visual circuit development. Elife 4, e10721

36. Hafner, M. and Koeppl, H. (2012) Stochastic simulations in systems biology. In Handbook of Research on Computational science and Engineering: Theory and Practice (Warrock, W., ed.), pp. 267-286, IGl Global

37. Korobkova, E. et al. (2004) From molecular noise to behavioural variability in a single bacterium. Nature $428,574-578$

38. Bruder, C.E. et al. (2008) Phenotypically concordant and discordant monozygotic twins display different DNA copy-number-variation profiles. Am. J. Hum. Genet. 82, 763-771

39. Maiti, S. et al. (2011) Ontogenetic de novo copy number variations (CNVs) as a source of genetic individuality: studies on two families with MZD twins for schizophrenia. PLoS One 6, e17125

40. Fraga, M.F. et al. (2005) Epigenetic differences arise during the lifetime of monozygotic twins. Proc. Natl. Acad. Sci. U. S. A. 102 10604-10609

41. Waddell, S. et al. (2014) Neural transposition in the Drosophila brain: is it all bad news? Adv. Genet. 86, 65-92

42. Lapp, H.E. and Hunter, R.G. (2016) The dynamic genome: transposons and environmental adaptation in the nervous system. Epigenomics 8, 237-249

43. Faulkner, G.J. and Garcia-Perez, J.L. (2017) L1 mosaicism in mammals: extent, effects, and evolution. Trends Genet. 33, 802-816

44. Vogt, G. et al. (2008) Production of different phenotypes from the same genotype in the same environment by developmental variation. J. Exp. Biol. 211, 510-523

45. Ayroles, J.F. et al. (2015) Behavioral idiosyncrasy reveals genetic control of phenotypic variability. Proc. Natl. Acad. Sci. U. S. A 112, 6706-6711

46. Tobin, W.F. et al. (2017) Wiring variations that enable and constrain neural computation in a sensory microcircuit. Elife 6, e24838

47. Enriquez, J. et al. (2018) Differing strategies despite shared lineages of motor neurons and glia to achieve robust development of an adult neuropil in Drosophila. Neuron 97, 538-554

48. Honegger, K. and de Bivort, B. (2018) Stochasticity, individuality and behavior. Curr. Biol. 28, R8-R12

49. Marder, E. et al. (2015) Robust circuit rhythms in small circuits arise from variable circuit components and mechanisms. Curr. Opin. Neurobiol. 31, 156-163

50. Kupiec, J.-J. (2008) L'Origine des Individus, Fayard 